\title{
Diagnóstico inmunológico y terapia del aborto recurrente espontáneo
}

\author{
Angela Cadavid, Beatriz Peña, Fabio Sánchez, Jorge Botero, Adriana de Castañeda, Jorge Ossa*
}

RESUMEN: Se presenta una recopilación del diagnóstico inmunológico y los resultados preliminares del tratamiento, en parejas con aborto recurrente espontáneo atendidas en el Programa de Reproducción de la Universidad de Antioquia, en el período comprendido entre febrero de 1991 y noviembre de 1995.

PALABRAS CLAVES: Aborto recurrente espontáneo, factores bloqueadores, anticuerpos antifosfolípidos, aloinmunoterapia, autoinmunoterapia.

SUMMARY: This article is a compilation of the immunological diagnosis and preliminary results of the treatment of couples with recurrent spontaneous abortion attending the Reprodution Program at the University of Antioquia during the period of February 1991 to November 1995.

KEY WORDS: Recurrent spontaneous abortion, blocking factors, antiphospholipid antibodies, alloimmunotherapy, autoimmunotherapy.

\section{Introducción}

El aborto habitual asociado con un factor inmunológico, conjuntamente con el aborto asociado a alteraciones endocrinas, cromosómicas, anatómicas y agentes infecciosos, representan el $40 \%$ de las perdidas recurrentes del embarazo. El $60 \%$ restante persisten en la categoría de causa desconocida.

Del factor inmunológico, se han reconocido dos tipos: el autoinmune caracterizado por la presencia de anticuerpos contra fosfolípidos cargados negativamente y el aloinmune por una deficiencia en el reconocimiento, por parte de la madre, de antígenos paternos expresados por el feto.

Los anticuerpos contra antígenos fosfolipídicos, pueden ser determinados por pruebas de coagulación dependientes de fosfolípidos (anticoagulante lúpico), o por inmunoensayo en fase sólida (anticuerpos antifosfolípidos). La presencia de estos autoanticuerpos, se ha asociado con trombosis arterial y venosa, trombocitopenia, livedo reticularis manifestaciones neurológicas y pérdidas fetales.

Los mecanismos patogénicos que conducen a la coagulación y trombosis, en el caso de los anticuerpos antifosfolípidos, permanecen desconocidos, pero se han postulado varias explicaciones: disfunción de la proteína C, inhibición de la liberación de prostaciclina endotelial, activación del complemento sérico y la inhibición de proteínas anticoagulantes placentarias. Adicionalmente, se ha postulado que los anticuerpos antifosfolípidos interfieren con la fusión del sincitiotrofoblasto a partir del citotrofoblasto llevando a una inadecuada formación de la placenta.

Programa Reproducción, Facultad de Medicina, Universidad de Antioquia, A.A. 1226, Medellín - Colombia
Para los trastornos autoinmunes se han propuesto diferentes esquemas terapéuticos tales como aspirina, heparina, prednisona o diferentes combinaciones de estas. También ha sido utilizada la inmunoglobulina intravenosa.

La deficiente respuesta aloinmune de la madre está enmarcada dentro de la hipótesis inmunotrópica, según la cual la madre debe reconocer los antígenos paternos que está expresando el feto y desarrollar una respuesta inmune, que no sólo no sea nociva, sino que además promueva el desarrollo y crecimiento de la unidad fetoplacentaria. La naturaleza del antígeno (o los antígenos) que estimulan esta respuesta materna, no ha sido aclarada y hay varios candidatos propuestos: $\mathrm{R} 80 \mathrm{~K}$, TLX, HLA-G.

La cuantificación de esta respuesta inmune materna es muy compleja y no existe un método diagnóstico que permita evaluarla de una manera precisa. Se han hecho algunas aproximaciones mediante la evaluación de los factores bloqueadores del cultivo mixto de linfocitos maternos contra linfocitos paternos; esta es una técnica in vitro que permite determinar la alo-reactividad de los linfocitos maternos a los linfocitos paternos, como un reflejo de la alo-reactividad in vivo a los antígenos que se expresan en trofoblasto y que al parecer también se expresan en sangre periférica. La otra posibilidad de bloqueo del cultivo mixto, que consideramos más plausible, es el bloqueo del receptor de las células $\mathrm{T}$ en las células efectoras maternas.

La terapia con linfocitos paternos y/o de donante (aloinmunoterapia) ha sido la más ampliamente utilizada para el aborto habitual de causa aloinmune, pero sus resultados son controvertidos. Recientemente, se realizó un Estudio Colaborativo Internacional, para tratar de resolver la controversia de la efectividad de la terapia. 
Este estudio mostró una diferencia significativa entre los nacidos vivos después de la terapia, en comparación con grupos controles no tratados $(60.7 \%$ vs $44.4 \%, p=0.006)$, con un beneficio absoluto de la terapia entre 8 y $10 \%$. Cuando se hizo el análisis en un grupo de pacientes con criterios más estrictos de selección con respecto al diagnóstico, se aumentó el beneficio absoluto del tratamiento a $16.3 \%$.

En conclusión, la eficacia de la terapia no pudo ser descartada ni confirmada con este estudio, pero se planteó la necesidad de desarrollar métodos diagnósticos más adecuados que permitan seleccionar las pacientes que se beneficiarían de la inmunoterapia.

En este trabajo se presenta un compendio del diagnóstico inmunológico realizado a las parejas atendidas en nuestro programa, y los resultados parciales del tratamiento, en el período comprendido entre febrero de 1991 y noviembre de 1995.

\section{Materiales y métodos}

\section{Pacientes}

Se estudiaron todas las parejas con diagnóstico de aborto a repetición (dos o más abortos con el mismo compañero); las cuales fueron remitidas de diferentes instituciones y médicos particulares; durante el período comprendido entre febrero de 1991 y octubre de 1995.

Todas las parejas, a su ingreso, fueron entrevistadas por un médico inmunólogo, quien hizo la evaluación y orientación inicial, explicó los procedimientos diagnósticos y llenó la información en un formato de historia clínica. Preferiblemente, la pareja debía tener la evaluación básica de su infertilidad, y en el programa sólo se estudió la patología auto y aloinmune para completar el estudio.

\section{Determinación de anticuerpos antifosfolípidos}

A las mujeres se les extrajo $10 \mathrm{ml}$ de sangre periférica en tubo seco, se separó el suero y se almacenó a $-20^{\circ} \mathrm{C}$ hasta procesar la muestra. Por el método de ELISA, descrito por Beer y cols., se determinaron anticuerpos IgG e IgM contra seis fosfolípidos cargado negativamente: cardiolipina $(\mathrm{CL})$, fosfatidilglicerol $(\mathrm{PG})$, fosfatidilserina (PS), fosfatidilinositol (PI), fosfatidiletanolamina (PE) y ácido fosfatídico (PA).

\section{Anticoagulante lúpico}

Una muestra de sangre periférica con citrato de sodio, fue utilizada para la determinación del anticoagulante lúpico. Se realizó por la técnica de tiempo parcial de tromboplastina activado (TPTa), de Organón Teknika ${ }^{\mathrm{R}}$, de acuerdo con las recomendaciones de la casa productora.

\section{Factores bloqueadores del cultivo mixto de linfocitos}

Se obtuvieron muestras de sangre periférica de los dos miembros de la pareja, se separaron los linfocitos sobre un gradiente de Ficoll Hypaque y se cultivaron en presencia de $20 \%$ de una rnezcla de suero humano o de suero de la paciente, inactivados. Los linfocitos de la mujer se tomaron como efectores y los del esposo, previamente tratados con mitomicina-C, como estimuladores. Después de 7 días de cultivo, se agregó timidina tritiada a una concentración de $0.5 \mathrm{uCi} /$ pozo/ $18 \mathrm{~h}$, se colectaron las células en filtros de fibra de vidrio, se agregó cocktail de centelleo y se midió la proliferación celular en cpm en un contador $\beta$

Se estableció el índice de proliferación con ambos sueros, se calculó el porcentaje de inhibición para determinar la presencia de factores bloqueadores de la proliferación. Valores por debajo de $30 \%$ de inhibición, se consideraron como negativos y se clasificó a la pareja como positiva para alteración aloinmune.

\section{Inmunizaciones}

A las parejas con alteración aloinmune, se les ofreció el tratamiento de inmunizaciones con linfocitos del esposo $y / o$ de un tercero. La pareja fue instruida para la selección del donante y firmaron un consentimiento escrito de las inmunizaciones. Tanto al esposo como al donante, se les realizó previamente VDRL, anticuerpos contra HIV y hepatitis $\mathrm{C}$ y el antígeno superficial de la hepatitis B.

Para las inmunizaciones, se tomaron $50 \mathrm{ml}$ de sangre heparinizada a cada uno de los donantes, se separaron Ios mononucleares y se aplicó cada suspensión de células en $1 \mathrm{ml}$ de solución salina, en tres inyecciones subcutáneas. El procedimiento se repitió dos veces con intervalo de 68 semanas antes de la gestación y se hicieron 1 ó 2 refuerzos durante el embarazo hasta la semana 20.

\section{Terapia anti-autoinmune}

A las parejas positivas para autoinmunidad (anticuerpos antifosfolípidos positivos y/o anticoagulante lúpico), se les prescribió $100 \mathrm{mg} /$ día de ácido acetil salicílico (aspirineta) desde el día 1 del ciclo hasta la semana 36 de la gestación. Dado los costos de la heparina, que no la hacen accesible a todas las pacientes, su uso se sugirió como opcional. La dosis recomendada fue 5.000 U.I s.c. cada 12 horas desde el día +1 del ciclo hasta la semana 34 de la gestación. Si no ocurrió el embarazo en ese ciclo, se suspendió desde el día de la menstruación hasta la ovulación en el ciclo siguiente.

A las pacientes que presentaron anticoagulante lúpico, se les prescribió además prednisona $10 \mathrm{mg} / 12 \mathrm{~h}$ desde el día 1 del ciclo hasta la semana 28 de la gestación, con disminución gradual desde la semana 24 .

\section{Terapia única}

Con la hipótesis de que los mecanismos patogénicos que llevan al aborto por alteración aloinmune y autoinmune son fundamentalmente los mismos, esto es, inflamación y trombosis, se propuso que la terapia única anti-autoinmune con la aspirina (antitrombótica y antiinflamatoria) y con heparina (anticoagulante), podía ser un tratamiento eficiente del ARE con ambas alteraciones.

Los criterios de selección de las parejas, además de presentar anticuerpos antifosfolípidos positivos y no tener factores bloqueadores del cultivo mixto de linfocitos, 
fueron: abortadoras primarias, edad de la mujer menor de 35 años, ninguna otra causa conocida de aborto. A todas las parejas que cumplieron con los requisitos, se les explicó en forma clara los objetivos del estudio y se les invitó a participar con el incentivo de recibir por parte del proyecto, la droga y el seguimiento inmunológico durante la gestación. Las parejas firmaron un consentimiento.

El esquema de terapia consistió de $100 \mathrm{mgs}$ de ácido acetil salicílico desde el día 10 . del ciclo hasta la semana 36 del embarazo y $5.000 \mathrm{U} / 12 \mathrm{~h}$ de heparina subcutánea desde el día 14 del ciclo, hasta la semana 34 . No se aplicaron inmunizaciones con linfocitos.

\section{Resultados}

\section{Diagnóstico de autoinmunidad}

En el Programa se atendieron 578 parejas con diagnóstico de aborto habitual, de las cuales 335 (58\%) tenían sólo 2 abortos y $243(42 \%)$ tenían 3 ó más abortos previos. A 286 de estas parejas se les realizó el diagnóstico de causa autoinmune por la determinación de los anticuerpos antifosfolípidos por ELISA (AFL) y por el anticoagulante lúpico (AL). De las 292 restartes sólo se pudo colectar información de anticuerpos antifosfolípidos.

La correlación de las dos pruebas, AFL y AL, no fue significativa y las pacientes positivas para ambas pruebas, fueron $25(8.8 \%)$; negativas para ambas, $140(49 \%)$; positivas para AL y negativas para AFL,29 (10\%); negativas para $\mathrm{AL}$ y positivas para $\mathrm{AFL}, 92$ (32.2\%).

E1 AFL más frecuentemente asociado con el AL fue cardiolipina (IgM), seguido en su orden por p-inositol (IgG), ácido fosfatídico (IgM), p-inositol (IgM). La asociación con cardiolipina IgG fue de sólo $7.4 \%$ y con la petanolamina $\operatorname{IgG}$, fue de 0 . No se encontró diferencia entre la concordancia de las pruebas y el número de abortos previos o el antecedente de un hijo vivo.

En general, se hizo diagnóstico de autoinmunidad positiva a $273 / 578(47 \%)$ de las parejas, de las cuales $5.8 \%$ tenían dos abortos y $42 \%$ tres o más.

Los fosfolípidos más frecuentes a los cuales las pacientes eran positivas fueron en su orden: IgG para $\mathrm{p}$ inositol, ácido fosfatídico, cardiolipina y p-glicerol. Para $\operatorname{IgM}$, se encontró mayor positividad para p-etanolamina y p-inositol.

La frecuencia de pacientes positivas para AFL, se resume en la siguiente tabla:

\begin{tabular}{|lrrrrrr|}
\hline & CL & PG & PA & PI & PE & PS \\
$\operatorname{IgG}(\%)$ & 10.8 & 10.4 & 12.0 & 12.0 & 7.5 & 7.2 \\
$\operatorname{IgM}(\%)$ & 4.2 & 5.5 & 5.2 & 6.3 & 6.8 & 3.5 \\
\hline
\end{tabular}

No se encontró asociación positiva entre el número de pérdidas y la positividad de los AFL, ni entre el número de pérdidas y el número de especificidades a las cuales se fuera positivo.

Con respecto al resultado de la nueva gestación, después de realizado el diagnóstico y prescrito la terapia, se encontró un efecto positivo de la misma; es decir, una diferencia significativa entre el porcentaje de nacidos vivos y el porcentaje de pérdidas en las pacientes que eran positivas para alguno de los siguientes anticuerpos:
IgG p-inositol, IgG p-glicerol, IgG cardiolipina, IgG ácido fosfatídico, IgM p-inositol e IgM p-glicerol.

\section{Diagnóstico de aloinmunidad}

De las 275 parejas a las cuales se les realizó el CML, $206(75 \%)$ fueron aloinmune positivas (64\% con 2 abortos y $.36 \%$ con 3 ó +) y $253(69 \%)$, fueron negativas (46\% con 2 abortos y $54 \%$ con 3 ó + ).

La distribución de las 578 parejas por diagnóstico inmunológico y por número de abortos previos fue la siguiente:

\begin{tabular}{|l|c|c|c|}
\hline & $\begin{array}{c}2 \\
\text { abortos }\end{array}$ & $\begin{array}{c}3 \text { ó }+ \\
\text { abortos }\end{array}$ & Total \\
\hline alo+/AFL+o AL+ & 75 & 41 & $116(20.1 \%)$ \\
alo+/AFL-y AL- & 44 & 21 & $65(11.2 \%)$ \\
alo+/AFL-y AL (NE) & 12 & 13 & $25(4.3 \%)$ \\
alo-/AFL+ o AL+ & 18 & 17 & $35(6.1 \%)$ \\
alo-/AFL-y AL- & 3 & 13 & $16(2.8 \%)$ \\
alo-/AFL-y AL (NE) & 11 & 7 & $18(3.2 \%)$ \\
alo(NE)/AFL+ o AL+ & 67 & 55 & $122(21.1 \%)$ \\
alo(NE)/AFL-y AL- & 35 & 26 & $61(10.5 \%)$ \\
alo(NE)/AFL-y AL (NE) & 70 & 50 & $120(20.7)$ \\
\hline
\end{tabular}

$\mathrm{NE}=$ no estudiado.

\section{Resultados de la nueva gestación en las pacientes acorde a su estado inmunológico}

De las 206 parejas aloinmunes positivas en las cuales estaba indicada las inmunizaciones, 151 han recibido la terapia con linfocitos, 12 parejas ingresaron al estudio de terapia única anti-autoinmune, 5 parejas no aceptaron la terapia y se embarazaron y las restantes no se han inmunizado todavía.

De las 151 parejas inmunizadas, 100 se han embarazado $\mathrm{y}$, al momento de este reporte, 79 han terminado su embarazo con los siguientes resultados: 55 (70\%) tuvieron un hijo vivo y $24(30 \%)$ presentaron un nuevo aborto. $\mathrm{p}<0.001$.

Los resultados de la nueva gestación según el perfil del estado inmunológico y la terapia prescrita son los siguientes:

\begin{tabular}{|l|c|c|c|c|}
\hline Estado inmune & Vivos & Pérdidas & Total & Valor $\mathrm{p}$ \\
\hline alo+/AFL+ o AL+ & 26 & 7 & 33 & $<0.01$ \\
alo+/AFL-yAL- & 22 & 14 & 36 & $\mathrm{P}=0.06$ \\
alo+/AFL-y LA(NE) & 7 & 3 & 10 & $\mathrm{p}=0.07$ \\
alo-/AFL+ o AL+ & 9 & 1 & 10 & $\mathrm{p}<0.01$ \\
alo-/AFL-y AL- & 8 & 6 & 14 & $\mathrm{p}=0.48$ \\
\hline
\end{tabular}

$\mathrm{NE}=$ no estudiado.

\section{Resultados de la terapia anti-autoinmune como único tratamiento del ARE en pacientes alo y autoinmune positivas}

Hasta la fecha, 12 parejas han ingresado al estudio de terapia única: 3 terminaron exitosamente su gestación con hijos vivos, 2 están en el último trimestre del emba- 
razo, 4 no se han embarazado aún y 3 , se retiraron del estudio por inconformidad con la heparina.

\section{Resultados de segundos embarazos en parejas después de recibir la terapia con linfocitos:}

Se tienen datos de algunas parejas que han embarazado por segunda vez después de la terapia con linfocitos. Los datos son muy preliminares, pero es interesante anotar que independientemente del resultado de la gestación en el primer embarazo posterapia, algunas pacientes han tenido un hijo vivo sin neeesidad de muevas inmunizaciones.

\section{Discusión}

En resumen, a 232 pacientes se les pudo hacer el estudio inmunológico completo y de ellas, $216(93 \%)$ tenían por lo menos un factor inmune positivo y $16(7 \%)$, fueron negativas.

Hasta el momento de este informe, 79 de las 216 pacientes positivas han terminado su embarazo. Estas 79 pacientes habían recibido la terapia inmunológica apropiada acorde con el diagnóstico (inmunizaciones con linfocitos solas o asociadas con aspirina y/o heparina, o la terapia con aspirina y aspirina solamente). El porcentaje de éxito en términos de un hijo vivo, fue del $72 \%$. De las 16 pacientes que fueron negativas, 14 se han embarazado y $8(57 \%)$ terminaron su embarazo exitosamente. Estas pacientes recibieron o no otras terapias a juicio del médico tratante.

Por razones éticas ha sido imposible establecer un control de pacientes con factor inmune positivo y sin terapia, por lo tanto el efecto real del tratamiento no podría determinarse en este tipo de estudio. Si tenemos en cuenta la literatura universal y muy particularmente el Estudio Colaborativo Internacional de la aloinmunoterapia, que indica un porcentaje de éxito espontáneo de aproximadamente $56 \%$, el efecto del tratamiento en nuestro programa sería sólo del $16 \%$, lo cual coincide con los resultados del mencionado estudio internacional.

Este pequeño efecto benéfico debe ser considerado en forma crítica. En primer lugar el resultado parece ser consistente a nivel internacional, lo que nos hace pensar que se trata de un efecto real pero es necesario cuestionar si este reducido efecto justifica la aplicación de una terapia, que no es totalmente inocua y de la cual el mayor porcentaje de pacientes no va a obtener utilidad. Es necesario investigar la posibilidad de definir factores pronósticos con el fin de someter a la terapia sólo a aquellas mujeres que tengan la mayor probabilidad de beneficiarse de la misma. Igualmente, no se debe ignorar el impacto social de la terapia inmunológica teniendo en cuenta el factor humano de las parejas que, fuera de este tratamiento, no tienen otra posibilidad de ayuda terapéutica.

\section{Agradecimientos}

A COLCIENCIAS por la financiación a través del proyecto 1115-070491 y del Programa de Formación de Recursos Humanos; al Departamento de Antioquia por su contribución al Programa de Reproducción; a la Universidad de Antioquia.

A los médicos e instituciones que nos han remitido parejas.

A las parejas por su valiosa colaboración.

\section{BIBLIOGRAFIA}

1. Stray-Pedersen B, Stray-Pedersen S. Etiologic factors and subsequent reproductive performance in 195 couples with a prior history of habitual abortion. Am. J. Obstet. Gynecol. 1984; 148: 140-146.

2. Beer AE. Immunological aspects of normal pregnancy and recurrent spontaneous abortion. Semin. Reprod. Endocrinol. 1988; 6(2): 163178.

3. Uribe Oscar. Síndrome antifosfolípido primario y pérdida fetal. En: Ossa JE, Sánchez F, Cadavid Angela (eds). Inmunología de la Reproducción. Ed. U. de A., 1993; 95-103.

4. Kwak JY, Gilman-Sachs A, Beaman KD et al. Auntoantibodies in women with primary recurrent spontaneous abortion of unknown etiology. J. Reprod. Immunol. 1992; 22: 15-31.
5. The recurrent miscarriage immunotherapy trialist group. Worldwide collaborative observational study and meta-analysis on allogenic immunotherapy for recurrent spontaneous abortion. Am. J. Reprod. Immunol. 1994; 32: 55-72.

6. Daya S, Gunby J and The recurrent miscarriage immunotherapy trialist group. The effectiveness of allogenic leukocyte immunization in unexplained primary recurrent spontaneous abortion. Am. J. Reprod. Immunol. 1994; 32: 294-302.

7. Suciu-Foca S, Rohowsky C, Kung P et al. Idiotype-like determinants on human T lymphocytes alloactivated in mixed lymphocyte culture. J. Exp. Med. 1982; 164: 490-500. 